\title{
DocOx (AIO-PK0106): a phase II trial of docetaxel and oxaliplatin as a second line systemic therapy in patients with advanced pancreatic ductal adenocarcinoma
}

Thomas J. Ettrich ${ }^{\dagger}$, Lukas Perkhofer ${ }^{\dagger}$, Goetz von Wichert ${ }^{2}$, Thomas M. Gress ${ }^{3}$, Patrick Michl ${ }^{4}$, Holger F. Hebart ${ }^{5}$, Petra Büchner-Steudel ${ }^{4}$, Michael Geissler ${ }^{6}$, Rainer Muche ${ }^{7}$, Bettina Danner ${ }^{7}$, Volker Kächele ${ }^{8}$, Andreas W. Berger ${ }^{1}$, Melanie Güthle ${ }^{1}$ and Thomas Seufferlein ${ }^{1 *}$

\begin{abstract}
Background: The current study was conducted to examine the activity of a docetaxel/oxaliplatin (DocOx) combination as second line treatment for advanced pancreatic ductal adenocarcinoma (Trial registration: NCT00690300. Registered June 2, 2008)

Methods: DocOx is a prospective, multi-center, single arm, phase II trial using docetaxel $\left(75 \mathrm{mg} / \mathrm{m}^{2}, 60 \mathrm{~min}, \mathrm{~d}\right.$ 1) and oxaliplatin ( $\left.80 \mathrm{mg} / \mathrm{m}^{2}, 120 \mathrm{~min}, \mathrm{~d} 2\right)$ in 21-day cycles. The treatment period was scheduled for up to 8 cycles. Primary endpoint was tumor response according to RECIST 1.0. Secondary endpoints were progression free survival, overall survival, safety/toxicity, quality of life and clinical benefit.
\end{abstract}

Results: Data represent the intention to treat analysis of 44 patients with chemorefractory pancreatic cancer enrolled between 2008 and 2012 at five institutions in Germany. The primary endpoint of tumor response was achieved in $15.9 \%$ of the patients (7 partial remissions, no complete remission), with a disease control rate of $48 \%$ after the first two treatment cycles. Median progression free survival (PFS) was 1.82 months (CI 95 \% 1.5-3.96 months) and median overall survival (OS) was 10.1 months (Cl $95 \%$ 5.1-14.1 months).

Conclusions: This single-arm trial demonstrates that the combination of docetaxel and oxaliplatin yields promising results for the treatment of advanced pancreatic ductal adenocarcinoma patients. Selected patients had particular benefit from this treatment as indicated by long PFS and OS times. Even after 8 cycles of treatment with DocOx a partial response was observed in 2 patients and stable disease was observed in another 6 patients. The data obtained with the DocOx protocol compare well with other second line protocols such as OFF (oxaliplatin, 5-FU, leucovorin). The DocOx regimen could be an interesting option for patients who received gemcitabine as first line treatment for metastatic pancreatic cancer.

Keywords: Pancreatic cancer, Advanced disease, Second line therapy

\footnotetext{
*Correspondence: thomas.seufferlein@uniklinik-ulm.de

${ }^{\dagger}$ Equal contributors

${ }^{1}$ Department of Internal Medicine I, Ulm University, Albert-Einstein-Allee 23,

D-89081 Ulm, Germany

Full list of author information is available at the end of the article
}

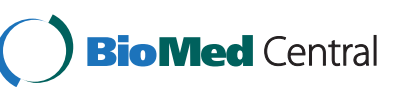

(C) 2016 Ettrich et al. Open Access This article is distributed under the terms of the Creative Commons Attribution 4.0 International License (http://creativecommons.org/licenses/by/4.0/), which permits unrestricted use, distribution, and reproduction in any medium, provided you give appropriate credit to the original author(s) and the source, provide a link to the Creative Commons license, and indicate if changes were made. The Creative Commons Public Domain Dedication waiver (http://creativecommons.org/publicdomain/zero/1.0/) applies to the data made available in this article, unless otherwise stated. 


\section{Background}

Pancreatic ductal adenocarcinoma (PDAC) is a major cause of cancer related deaths in the Western world. The only curative option for PDAC is surgery, but at the time of primary diagnosis only $10-15 \%$ of patients are eligible for surgery with curative intent. The main limitation is the delayed diagnosis at an already locally advanced or metastatic state of the disease $[1,2]$. Consequently, systemic therapy is the treatment of choice for the majority of patients. The standard of care in this setting has developed over the last decade. FOLFIRINOX and the combination of gemcitabine/nabpaclitaxel have proven to be superior to single agent gemcitabine in the first-line therapy of metastatic PDAC $[3,4]$. Second line strategies in PDAC achieve a median progression free survival (mPFS) of 4 months and a median overall survival (mOS) of 6 months, respectively [5]. However, the optimal second-line strategy for PDAC still remains to be defined [6,7]. Compared to best supportive care (BSC) or 5-fluorouracil (5-FU) alone the combination of 5-FU, leucovorin and oxaliplatin (OFF) significantly prolonged the overall survival time in ECOG 0-2 (Eastern Cooperative Oncology Group) patients $[8,9]$. Recently, the combination of nanoliposomal irinotecan plus 5-FU has also shown superiority as second line treatment for PDAC compared to 5-FU alone (mPFS 3.1 versus 1.5 months, HR 0.56 ; mOS 6.1 versus 4.2 months, HR 0.67) [10]. The objective response rate is generally low in the second line setting [11]. Single agent docetaxel achieves response rates of up to $15 \%$ as first line therapy of advanced PDAC $[12,13]$, and has moderate activity as second line treatment of PDAC in retrospective analyses [14, 15]. Oxaliplatin-based combination regimen show similar response rates as docetaxel [16-18]. Several phase I/II studies confirmed the efficacy and safety of the combination of docetaxel plus oxaliplatin for different tumor entities [19-21]. To date the combination of both substances has not been evaluated in the treatment of chemorefractory PDAC. The current study was conducted to prospectively evaluate the activity and feasibility of the combination of docetaxel/oxaliplatin (DocOx) as second line treatment of PDAC.

\section{Patients and methods}

The DocOx trial (NCT00690300) was designed as an open label, multicenter, single arm, phase II study. Between February 2008 and March 2012, 47 patients were enrolled at five German institutions. The final analysis was restricted to 44 patients.

\section{Patient population}

Inclusion criteria were as follows: histologically or cytologically confirmed metastatic or unresectable locally advanced PDAC; age $\geq 18$ years; at least one measurable target lesion according to RECIST 1.0 (Response Evaluation Criteria in Solid Tumors) outside any previously irradiated area; failure of first line therapy of metastatic or unresectable locally advanced PDAC due to progressive disease during or within 3 months after finishing first line chemotherapy; Karnofsky performance score (KPS) > 60 \% (ECOG $0-2)$; life expectancy $\geq 12$ weeks; adequate bone marrow function (granulocyte count $\geq 1.5 \times 10^{9} / \mathrm{L}$, platelet count $\geq 100 \times 10^{9} / \mathrm{L}$, hemoglobin $\geq 9 \mathrm{~g} / \mathrm{dl}$ ); serum bilirubin levels $<2$ times upper limit of normal (ULN), up to 2.5 times ULN in case of hepatic metastasis (biliary drainage allowed); transaminases $<2.5$ times ULN.

Exclusion criteria were as follows: Any other primary tumor or secondary malignancy except basal cell carcinoma of the skin or in situ carcinoma of the cervix uteri (patients with adequately treated other malignancies and tumor absence for $\geq 5$ years were eligible); pregnancy or breastfeeding period; patients unable to ensure adequate contraception; known cerebral metastasis; uncontrolled severe infections; peripheral neuropathy exceeding CTCAE (Common Terminology Criteria for Adverse Events) grade 1.

All patients signed a written informed consent according to national and local regulations. The protocol was approved by the Ethics Committee of Ulm University [22].

\section{Treatment plan}

In this open label trial patients received docetaxel $75 \mathrm{mg} / \mathrm{m}^{2}$ (60 min iv infusion) on day 1 and oxaliplatin $80 \mathrm{mg} / \mathrm{m}^{2}$ (120 min iv infusion) on day 2 , repeated every 3 weeks. Treatment was administered at least for 8 cycles, unless there was tumor progression, unacceptable toxicity or patient refusal. In case of stable disease (SD) after 8 cycles patients could choose to carry on with the therapy. Premedication included adequate antiemetic therapy and oral dexamethasone $8 \mathrm{mg}$ the day prior to docetaxel application as well as on days 2 and 3 after docetaxel treatment. On treatment days the patients received another $16 \mathrm{mg}$ of dexamethasone iv. To prevent oxaliplatin related polyneuropathy $1 \mathrm{~g}$ calcium gluconate and $1 \mathrm{~g}$ magnesium gluconate were administered iv prior to and after oxaliplatin infusion. In case of severe hematotoxicity prophylactic granulocyte colony-stimulating factor treatment in addition to dose modification was applied. In case of neutropenia $<1.5 \times 10^{9} / \mathrm{L}$, thrombopenia $<100 \times 10^{9} / \mathrm{L}$, diarrhea $>$ grade 1 , peripheral neuropathy $>$ grade 1 or other nonhematologic toxicities > grade 1 treatment could be delayed up to a maximum of 2 weeks. In case of a 2 weeks delay and ongoing neutropenia the dose of both cytostatics was reduced to $50 \%$ if neutrophiles were 1.0 to $1.5 \times 10^{9} / \mathrm{L}$ or if platelets were 50 to $100 \times 10^{9} / \mathrm{L}$. Treatment was discontinued in case of neutropenia $<1.0 \times 10^{9} / \mathrm{L}$, thrombopenia $<50 \times 10^{9} /$ 
L, peripheral neuropathy grade 2 or other ongoing nonhematologic toxicities after 2 weeks delay. The dose of both drugs was reduced to $80 \%$ if granulocytes were less than $<0.5 \times 10^{9} / \mathrm{L}$ or $<1.0 \times 10^{9} / \mathrm{L}$ with fever exceeding $38.5{ }^{\circ} \mathrm{C}$, if platelets were $<50 \times 10^{9} / \mathrm{L}$ or in case of nonhematologic toxicity > grade 2 (except alopecia, nausea and vomiting). In case of peripheral neuropathy grade 2 oxaliplatin was reduced to $50 \%$ and docetaxel to $80 \%$ of the previous dose level after recovery. Toxicity was assessed using the National Cancer Institute (NCI) common toxicity criteria (CTC) version 3 . Repeated severe toxicity after the second dose adjustment resulted in termination of the treatment.

\section{Pretreatment evaluation and follow-up}

The baseline evaluation included a complete medical history, physical examination including vital signs, an electrocardiogram (ECG), complete blood count (CBC) plus serum chemistry and, in case of child-bearing age, a pregnancy test, all within one week prior to start of treatment. A chest $\mathrm{x}$-ray and abdominal computed tomography were required to define the target lesion(s). All patients received questionnaires to assess quality of life (EORTC QLQ-C30) and clinical benefit (pain, use of analgesics, body weight, Karnofsky performance score) prior to each treatment cycle, furthermore the clinical benefit was recorded weekly within each cycle. Assessments before start and then weekly within each cycle included physical examination, CBC plus serum chemistry and recording of adverse events. Tumor response was evaluated by computed tomography after every second cycle according to RECIST 1.0 for the defined target lesions. Planned study termination after 8 cycles was followed by six- weekly examinations including assessment of life status, physical examination and Karnofsky performance score. The treatment was stopped in case of progressive disease, inacceptable toxicity, incompliance, or patient's wish.

\section{Treatment evaluation}

The primary endpoint of the study was defined as tumor response according to RECIST 1.0. Toxicities were graded according to NCI CTC version 3.0 Severe adverse events (SAE) were defined as follows: any reaction, side effect or disease displaying an increased risk or danger for the patient. Quality of life was assessed based on the EORTC QLQ-C30. Moreover, clinical benefit (CB) was recorded in all patients (Additional file 1).

\section{Statistical analysis}

Tumor response was defined as the primary endpoint of the study. Secondary endpoints included PFS, OS, quality of life and clinical benefit. All patients treated for at least one cycle of chemotherapy, even in case of protocol violation, were included into final analysis on an intention to treat (ITT) basis. The trial was based on a Simon's two-stage design [23]. For the sample size calculation a response rate of $\geq 15 \%$ was considered sufficient in an interim analysis, whereas a rate $\leq 5 \%$ was insufficient. The size of the type I ( $\alpha$ ) and II ( $\beta$ ) errors were 0.1 and 0.2 , respectively. An interim analysis was planned after 22 patients and in case of no response the study would be closed prematurely. Otherwise another 22 patients were to be enrolled until the total number of 44 participants was reached. OS and PFS were estimated using the Kaplan-Meier method. A descriptive data analysis was done for EORTC-QLQ-C30 and clinical benefit. The clinical benefit was calculated from four parameters (painintensity, use of pain-medication, KPS and body-weight) [24]. Pain intensity/use of analgetics and KPS were defined as primary indicators, body weight counted as a secondary indicator. For the evaluation of the clinical benefit patients had either to be positive, stable or negative classified for the primary indicators. Only in case of stable primary indicators the secondary indicator, body weight, was included for overall assessment. For positive evaluation of the clinical benefit at least 4 weeks of improvement of the indicators were required. A diagram for the assessment of the clinical benefit is displayed in the (Additional file 2).

\section{Results \\ Patients characteristics}

A total of 47 patients were recruited between February 2008 and March 2012 at five German institutions. The primary analysis was restricted to 44 patients (ITTpopulation). Three patients did not start treatment due to death (two patients) or refusal (one patient). The first stage of the study included 22 patients for interim analysis. After fulfilling the preset requirements for proceeding of the trial (response rate $\geq 15 \%$ ) another 22 patients were enrolled. The patient characteristics are listed in Table 1. As first line treatment all patients received a gemcitabine-based regimen, except two patients who received a 5-FU based concept. The median duration of first line therapy was 4.5 months (2.1-7.25 months). The main reasons for discontinuation of first line therapy were progressive disease in 42 patients (95.5\%) and toxicity in two cases $(4.5 \%)$. Most of the patients $(81.8 \%, 36 / 44)$ had metastatic disease at initiation of second line therapy.

\section{Dose intensity and efficacy}

In median 3 weeks passed between termination of the first line therapy and start of the second line treatment. From the ITT-population four patients $(9.1 \%)$ received only 1 cycle, another twelve $(27.3 \%)$ only 2 cycles, respectively. The median number of chemotherapy cycles was 4 (range: 1-8 cycles). Nine 
Table 1 Baseline Characteristics

\begin{tabular}{|c|c|c|}
\hline \multicolumn{3}{|l|}{ Docetaxel/Oxaliplatin $(n=44)$} \\
\hline Patient Characteristics & $\begin{array}{l}\text { Number of } \\
\text { Patients }\end{array}$ & Percent \\
\hline \multicolumn{3}{|l|}{ Sex } \\
\hline Male & 29 & 65.9 \\
\hline Female & 15 & 34.1 \\
\hline \multicolumn{3}{|l|}{ Age (years) } \\
\hline Median (and range) & 66.5 & $38-76$ \\
\hline \multicolumn{3}{|c|}{ Karnofsky performance status score $(n=43)$} \\
\hline $100 \%$ & 9 & 20.9 \\
\hline $90 \%$ & 21 & 48.8 \\
\hline $80 \%$ & 13 & 30.2 \\
\hline \multicolumn{3}{|l|}{ Prior surgery } \\
\hline No & 26 & 59 \\
\hline Curative Intention & 9 & 20.5 \\
\hline Palliative & 9 & 20.5 \\
\hline \multicolumn{3}{|l|}{ Prior radiotherapy } \\
\hline Yes & 3 & 6.8 \\
\hline No & 41 & 93.2 \\
\hline \multicolumn{3}{|l|}{ Location of the primary } \\
\hline Head & 26 & 59.1 \\
\hline Body & 10 & 22.7 \\
\hline Tail & 8 & 18.2 \\
\hline \multicolumn{3}{|l|}{ Disease extension } \\
\hline Locally advanced & 8 & 18.2 \\
\hline Metastatic & 36 & 81.8 \\
\hline \multicolumn{3}{|l|}{ Metastatic sites ${ }^{a}$} \\
\hline Liver & 30 & 68.1 \\
\hline Lymphnodes & 10 & 22.7 \\
\hline Lung & 7 & 15.9 \\
\hline Bone & 2 & 4.5 \\
\hline $\begin{array}{l}\text { Median duration of first line } \\
\text { therapy (mts) }\end{array}$ & 4.5 & $95 \%$ Cl 2.1-7.25 \\
\hline
\end{tabular}

patients (20.5\%) completed the pre-planned 8 cycles of chemotherapy. Ten patients were eligible for final staging by computed tomography after 8 cycles: two had continuous partial response (PR), six stable disease (SD) and two progressive disease (PD). The final analysis includes one patient with a total of 7 cycles chemotherapy who refused the last cycle. Interestingly, even after 8 cycles of treatment with DocOx, a partial response was observed in two patients and stable disease in another six patients corresponding a disease control rate of $18 \%$.

The main reason for discontinuation of treatment was PD in 28 cases (63.6\%) Toxicity and death were in charge for therapy discontinuation each within three cases $(6.8 \%)$. Seven patients $(15.9 \%)$ completed the planned treatment of 8 cycles and four continued therapy beyond the planned treatment period. The median relative dose intensity for both drugs was $95.7 \%$ of the theoretical dose for the applied cycles. The dose intensity was slightly higher for docetaxel compared to oxaliplatin (97.5\% vs. $93.5 \%$ ).

All patients had at least one measurable lesion for response assessment. Seven patients exhibited a partial response according to RECIST 1.0 (15.9\%, $95 \%$ CI 10-26\%, see Table 2). Stable disease was observed in 14 patients $(31.8 \%)$. The calculated disease control rate (DCR) was $47.7 \%$. The DCR was defined as the proportion of patients with $\mathrm{PR}$ or $\mathrm{SD}$ for at least 2 cycles. The median PFS was 1.82 months (CI $95 \%$ 1.5-3.96 months), Fig. 1. The PFS rate at 6 months and 1 year was $17.1 \%$ in both cases. The median OS was 10.1 months (CI $95 \%$ 5.1-14.1 months), Fig. 2. OS rates were $56.8 \%$ at 6 months and $39.3 \%$ at 1 year, respectively. There is one exceptional long time survivor with an overall survival of 75 months from primary diagnosis and a PFS of 36 months after start of second line treatment with a total of 22 cycles of docetaxel/oxaliplatin.

\section{Quality of life and clinical benefit}

Quality of life (QoL) was assessed using the QLQC30 questionnaire. A descriptive analysis revealed relevant changes in quality of life. Interestingly, QoL was independent from therapy response. The clinical benefit was calculated from the four parameters painintensity, use of analgesics, KPS and body-weight according to Burris et al. [24] and as described above. Five patients $(11.4 \%)$ described a clinical benefit (two patients with a tumor response and three without

Table 2 Response and Survival

\begin{tabular}{lll}
\hline Docetaxel/Oxaliplatin $(n=44)$ & & \\
\hline Efficacy & Number of Patients & Percent \\
\hline Response & & \\
$\quad$ Complete response (CR) & 0 & 15.9 \\
Partial response (PR) & 7 & 31.8 \\
Stable disease (SD) & 14 & 52.3 \\
Progressive disease (PD) & 23 & 47.7 \\
Disease control rate & 21 & \\
(CR + PR + SD) & & $95 \% \mathrm{Cl}$ \\
Survival & months & $1.5-3.96$ \\
$\quad$ Median progression & 1.82 & \\
free survival & & $5.1-14.1$ \\
Median overall survival & 10.1 & \\
\hline
\end{tabular}

$95 \% \mathrm{Cl}$ confidence interval 


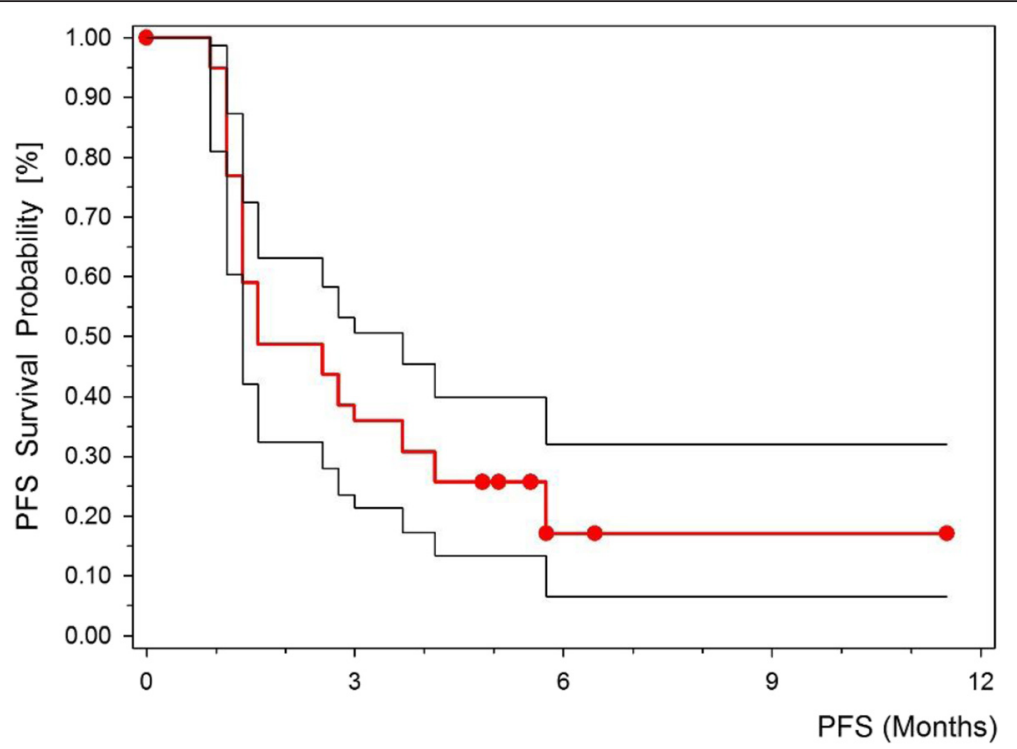

Fig. 1 Kaplan-Meier plot: second line progression free survival time with $95 \%$ confidence interval. PFS progression free survival

response) at different time points of study termination. In 39 cases $(88.6 \%)$ the score worsened. The $\mathrm{CB}$ parameters are listed in Table 3. Pain intensity was stable or decreased in the majority of patients $(90.2 \%, 37 / 41)$ throughout the therapy. The KPS was stable in 28 patients $(63.6 \%)$ during the course of treatment. Any loss of body weight was noticed in 40 patients $(90.9 \%)$.

From the collected data for Global Health Status out of the EORTC QLQ-C30 questionnaire we calculated the median time until definitive deterioration (TUDD). The TUDD was calculated in accordance to the published papers of Anota et al. and Bonnetain et al. and defined as an ongoing deterioration of at least five points as compared to the baseline $[25,26]$. The median TUDD was calculated with 3.5 months.

Safety

Treatment had to be discontinued in three patients (6.8\%) due to hematologic toxicity. Table 4 summarizes

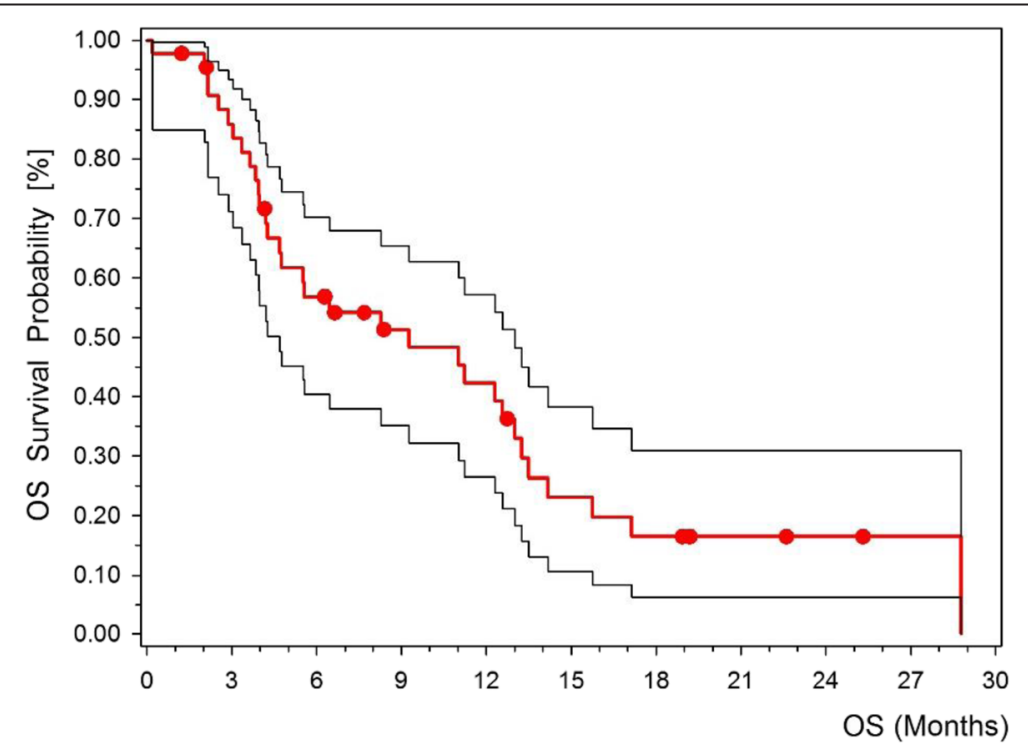

Fig. 2 Kaplan-Meier plot: second line overall survival time with $95 \%$ confidence interval. OS overall survival 
Table 3 Clinical benefit

\begin{tabular}{|c|c|c|c|}
\hline \multicolumn{4}{|c|}{ Docetaxel/Oxaliplatin $(n=44)$} \\
\hline & Number of Patients & Percent & $95 \% \mathrm{Cl}$ \\
\hline Clinical benefit response & 5 & 11.4 & $3.79-24.56$ \\
\hline \multicolumn{4}{|l|}{ Pain intensity $(n=41)$} \\
\hline Decreased & 4 & 9.8 & $2.72-23.13$ \\
\hline Stable & 29 & 70.7 & $54.46-83.87$ \\
\hline Improved & 8 & 19.5 & $8.82-34.87$ \\
\hline \multicolumn{4}{|c|}{ Karnofsky Perfomance Score } \\
\hline Decreased & 16 & 36.4 & $22.41-52.23$ \\
\hline Stable & 28 & 63.6 & $47.77-77.59$ \\
\hline \multicolumn{4}{|l|}{ Body weight } \\
\hline Decreased & 40 & 90.9 & 78.33-97.47 \\
\hline Increased & 4 & 9.1 & $2.53-21.67$ \\
\hline
\end{tabular}

the most frequent adverse events. Neutropenia grade 3 to 4 occurred in $63.6 \%(28 / 44)$ of patients. Febrile neutropenia grade 3 to 4 was reported in $4.5 \%(2 / 44)$ of patients. However, only two patients $(4.5 \%)$ required granulocyte-colony stimulating factor (G-CSF) at least once during treatment. The major non-hematologic grade 3 or 4 adverse events were diarrhea $(11.4 \%, 5 / 44)$ and nausea $(9.1 \%, 4 / 44)$. Grade 1 or 2 peripheral neuropathy was reported in $52.3 \%(23 / 44)$ of patients, $\geq$ grade 3 in only one patient $(2.3 \%)$. The most common grade 1 or -2 toxicities were alopecia $(68.2 \%, 30 / 44)$ and mucositis $(29.3 \%, 13 / 44)$. A more detailed overview of the toxicities is shown in the (Additional file 1: Table S1). No unexpected toxicities were reported.

\section{Discussion and conclusions}

An increasing number of patients with PDAC are eligible for a second line therapy. A recently published

Table 4 Common Grade 3 or 4 Adverse Events

\begin{tabular}{lll}
\hline Docetaxel/Oxaliplatin $(n=44)$ & & \\
\hline Adverse Event & Number of Patients & Percent \\
\hline Hematologic & 28 & 63.6 \\
Neutropenia & 2 & 4.5 \\
Febrile Neutropenia & 1 & 2.3 \\
Thrombocytopenia & 1 & 2.3 \\
Anaemia & & \\
Non Hematologic & 2 & 4.5 \\
Fatigue & 5 & 11.4 \\
Diarrhea & 4 & 9.1 \\
Nausea & 1 & 2.3 \\
Peripheral Neuropathy & 2 & 4.5 \\
G-CSF use &
\end{tabular}

G-CSF granulocyte-colony stimulating factor systematic review found beneficial effects for second line chemotherapies compared to best supportive care in PDAC, in particular for combinations of platinum agents and fluorouracil or gemcitabine [5]. However, there is currently no standard of care in the second line setting in PDAC. Evidence is mainly based on few small phase II trials and one phase III trial [8, 9, 18, 27, 28]. The CONKO-003 trial demonstrated that the combination of oxaliplatin, 5-FU and leucovorin according to the OFF regimen extends the duration of overall survival compared to 5 -FU alone or to best supportive care $[8,9]$.

This phase II trial was conducted to establish the efficacy and safety of the combination of docetaxel and oxaliplatin in the second line setting and its impact on the quality of life and clinical benefit for patients with advanced, chemorefractory PDAC. Both substances have shown interesting response rates in the first $[12,13]$ and second line [14, 15] setting in metastatic PDAC as single agents or in combination. Being aware of the limitations of a single arm trial the combination of docetaxel/oxaliplatin achieved a response rate of $15.9 \%$, a DCR of $47.7 \%$, a median OS of 10.1 months (CI $95 \%$ 5.1-14.1 months) and a median PFS of 1.82 months (CI $95 \%$ 1.5-3.96 months). These data are comparable to other published protocols such as OFF (see Table 5). Interestingly, even after 8 cycles of treatment with DocOx, a partial response was observed in two patients and stable disease in another six patients corresponding to a disease control rate of $18 \%$. There is one exceptional long time survivor with an overall survival of 75 months from primary diagnosis and a PFS of 36 months after start of second line treatment with a total of 22 cycles of docetaxel/oxaliplatin that is still alive.

In our study the combination of docetaxel/oxaliplatin was in general well tolerated and no unexpected toxicities occurred during therapy. Most subjects experienced at least one grade $3 / 4$ adverse event, mainly hematological (neutropenia, $63.6 \%$ ) and diarrhea (11.4\%) see Table 4. Compared to the OFF regimen $[8,9]$, the DocOx protocol appears to be more toxic. However, all toxicities were manageable. 15 patients (34.1\%) were still eligible to third line therapy after progress to DocOx, see Table 6. The rate of febrile neutropenia was low $(<5 \%)$ and only two patients required single doses of G-CSF. A calcium and magnesium infusion was applied at each cycle because at the time the trial was conducted this was supposed to prevent oxaliplatin-associated polyneuropathy [29]. However, new data refute this concept [30].

There are few data on quality of life and clinical benefit during second line treatment of patients with PDAC. For the assessment of clinical benefit we used a composite score of pain and analgesics requirements, Karnofsky performance status, and body weight. In total five patients 
Table 5 Second line therapies in advanced PDAC

\begin{tabular}{|c|c|c|c|c|c|c|c|c|}
\hline Regimens & Phase & Patients & mPFS (ms) & mOS (ms) & OS 6-ms \% & DCR \% & PR \% & Ref \\
\hline FOLFIRINOX & Ret & 22 & 5.4 & 8.5 & n.a. & 63 & 19 & [32] 2011 \\
\hline FOLFIRINOX & Ret & 18 & 2.8 & 8.4 & 44.4 & 55.6 & 27.8 & [33] 2013 \\
\hline Nab-Paclitaxel & $\|$ & 19 & 1.7 & 7.3 & 58 & 37 & 5 & [31] 2012 \\
\hline FOLFIRI & $\|$ & 50 & 3.2 & 5.0 & 32 & 36 & 8 & [18] 2012 \\
\hline Capecitabine/Docetaxel & $\|$ & 43 & 3.7 & 5.3 & n.a. & 73 & 14 & [34] 2014 \\
\hline mFOLFOX & $\|$ & 30 & 1.5 & 3.7 & 30 & 17 & 7 & [28] 2009 \\
\hline FOLFIRI & & 31 & 2.1 & 4.2 & 27 & 23 & 0 & \\
\hline OFF & III & 23 & n.a. & 4.8 & n.a. & n.a. & n.a. & [8] 2011 \\
\hline BSC & & 23 & & 2.3 & & & & \\
\hline OFF & III & 76 & 2.9 & 5.9 & n.a. & n.a. & n.a. & [9] 2014 \\
\hline FF & & 84 & 2.0 & 3.3 & & & & \\
\hline Xelox & $\|$ & 39 & 2.5 & 5.8 & 44 & 28.6 & 2.6 & [35] 2008 \\
\hline Docetaxel & Ret & 17 & 2 & 4 & n.a. & 35 & 6 & [15] 2010 \\
\hline Nal-Iri & $\|$ & 40 & 2.4 & 5.2 & 42.5 & 50 & 7.5 & [27] 2013 \\
\hline Nal-lri/5-FU/LV & III & 417 & 3.1 & 6.1 & n.a. & n.a. & n.a. & [10] 2014 \\
\hline 5-FU/LV & & & 1.5 & 4.2 & & & & \\
\hline GEMOX & $\|$ & 33 & 4.2 & 6 & n.a. & 61.3 & 22.6 & [36] 2006 \\
\hline Vatalanib & $\|$ & 67 & 2 & n.a. & 29 & 31 & 3 & [37] 2014 \\
\hline DocOx & $\|$ & 44 & 1.8 & 10.1 & 56.8 & 48 & 15.9 & \\
\hline
\end{tabular}

mPFS median progression free survival time, mOS median overall survival time, Ref reference, Ret Retrospective, $m s$ months, $D C R$ disease control rate, $P R$ partial remission, ms months, OFF oxaliplatin, folinic acid, fluorouracil, LV leucovorine, 5-FU 5-fluorouracil, Nab-Paclitaxel nanoalbumine bound pacitaxel, Nal-Iri nanoliposomal irinotecan, FOLFIRI fluorouracil, leucovorine, irinotecan, FOLFOX fluorouracil, leucovorine, oxaliplatin, FOLFIRINOX fluorouracil, leucovorine, irinotecan, oxaliplatin, DocOx docetaxel oxaliplatin, n.a. not applicable, Ref Reference and publication date

(11.4\%) reported a clinical improvement at final examination. Cross trial comparisons to other second line trials are difficult, due to different chemotherapy regimens, heterogeneous patient collectives, distinct definitions of clinical benefit and mostly due to the fact that only few data are available. Recently, comparable results with an improvement of clinical benefit in $20 \%$ of patients were reported in a phase II trial using nanoliposomal-irinotecan as a single agent [27]. The median time until definitive deterioration of the Global health status was calculated with 3.5 months and is comparable to data from first line settings published so far [25].

The major limitation of our data is the single arm design. However, there was no established second line

Table 6 Third line therapies after failure of Docetaxel/Oxaliplatin treatment

\begin{tabular}{lll}
\hline Third line therapy $(n=15)$ & Number of Patients & Percent \\
\hline Treatment & 8 & 53.3 \\
\hline Gemcitabine + Capecitabine & 3 & 20 \\
5-FU/Oxaliplatin based & 4 & 26.7 \\
5-FU/Irinotecan based &
\end{tabular}

5-FU/Oxaliplatin based (OFF, FUFOX, XELOX, FOLFOX); 5-FU/Irinotecan based (XELIRI, FOLFIRI) chemotherapy available when this trial was initiated. Furthermore, similar trials confirmed that patients eligible for a second line treatment do not agree to be randomized to best supportive care only [8]. The data of this study compare well with those obtained with other protocols including the OFF regimen (see Table 5) and make this combination an option for patients with chemorefractory PDAC.

A second limitation is the fact that by now a substantial number of patients will have received oxaliplatin or a nabpaclitaxel in the first line setting due to the increased use of FOLFIRINOX or the gemcitabine/nab-paclitaxel regimen. However, a significant number of patients will still receive gemcitabine $+/$ - erlotinib in the first line setting and could benefit from docetaxel/oxaliplatin as second line treatment. Moreover, patients with an early relapse after adjuvant gemcitabine therapy who are not eligible for FOLFIRINOX maybe candidates for DocOx. In particular, the acceptable safety profile and the promising data on efficacy, quality of life and clinical benefit make this combination an interesting option for patients with chemorefratory pancreatic cancer. Recently, single agent nab-paclitaxel (phase II) [31] and the combination of nano-liposomal irinotecan/ 5-FU/ LV (phase III) [10] demonstrated promising results in this setting. It remains 
to be elucidated whether modern formulations of taxanes or irinotecan are superior to docetaxel in this setting.

\section{Additional files}

Additional file 1: Table S1. Adverse Events independent from relation to therapy. (DOCX $26.2 \mathrm{~kb}$ )

Additional file 2: Figure S1. Consort diagram. Figure S2. Flow chart for the assessment of the clinical benefit. (DOCX $82.8 \mathrm{~kb}$ )

\section{Abbreviations}

BSC: best supportive care; CB: clinical benefit; CBC: complete blood count; Cl: confidence interval; CTC: common toxicity criteria; CTCAE: Common Terminology Criteria for Adverse Events; DCR: disease control rate; DocOx: docetaxel/oxaliplatin; ECG: electrocardiogram; ECOG: Eastern Cooperative Oncology Group; EORTC: European Organization for Research and Treatment of Cancer; 5-FU: 5-fluorouracil; FOLFIRINOX: fluorouracil leucovorine, irinotecan, oxaliplatin; G-CSF: granulocyte-colony stimulating factor; HR: hazard ratio; ITT: intention to treat; KPS: karnofsky performance score; (m)OS: (median) overall survival; (m)PFS: (median) progression free survival; NCl: National Cancer Institute; OFF: 5-FU leucovorin, oxaliplatin; PD: progressive disease; PDAC: pancreatic ductal adenocarcinoma; PR: partial response; QLQ-C30: quality of life questionnaire-core 30; QoL: quality of life; RECIST: Response Evaluation Criteria in Solid Tumors; SAE: severe adverse events; SD: stable disease; TUDD: time until definitive deterioration; ULN: upper limit of normal.

\section{Competing interests}

The authors declare that they have no competing interests.

\section{Authors' contributions}

TJE, LP, MG, AWB, BD, RM have made substantial contributions to analysis and interpretation of data. TS, GW, VK have made substantial contributions conception and design of the study. MG, PM, TMG, HFH, PBS have made substantial contributions in the acquisition of data. TJE, LP, RM, TS have been involved in drafting the manuscript. All authors gave final approval for the version to be published.

\section{Acknowledgements}

Special thanks to Beate Einsiedler of the Institute of Epidemiology and Medical Biometry, Ulm University for statistical support. The final results were presented at the ASCO Gl Cancer Symposium 2015 where the abstract was awarded with a Merit Award by the Conquer Cancer Foundation of ASCO.

\section{Funding}

The trial was supported by Sanofi-Aventis.

\section{Author details}

'Department of Internal Medicine I, Ulm University, Albert-Einstein-Allee 23, D-89081 Ulm, Germany. ${ }^{2}$ Department of Internal Medicine, Schön-Klinik Hamburg-Eilbeck, Hamburg, Germany. ${ }^{3}$ Department of Gastroenterology, Endocrinology, Metabolism and Infectiology, Philipps University of Marburg, Marburg, Germany. ${ }^{4}$ Department of Internal Medicine I, Martin-Luther-University, Halle (Saale), Germany. ${ }^{5}$ Department of Internal Medicine, Stauferklinikum Schwaebisch-Gmuend, Mutlangen, Germany. ${ }^{6}$ Department of Internal Medicine, Oncology/Hematology, Gastroenterology, Esslingen Hospital, Esslingen, Germany. ${ }^{7}$ Institute of Epidemiology and Medical Biometry, Ulm University, Ulm, Germany. ${ }^{8}$ Praxis für Hämatologie und Onkologie UIm, Ulm, Germany.

Received: 28 July 2015 Accepted: 6 January 2016 Published online: 15 January 2016

\section{References}

1. Haberland J, Bertz J, Wolf U, Ziese T, Kurth BM. German cancer statistics 2004. BMC Cancer. 2010;10:52.

2. Jemal A, Siegel R, Xu J, Ward E. Cancer statistics, 2010. CA Cancer J Clin. 2010;60:277-300.
3. Conroy T, Desseigne F, Ychou M, Bouche O, Guimbaud R, Becouarn Y, et al. FOLFIRINOX versus gemcitabine for metastatic pancreatic cancer. N Engl J Med. 2011;364:1817-25.

4. Von Hoff DD, Ervin T, Arena FP, Chiorean EG, Infante J, Moore M, et al. Increased survival in pancreatic cancer with nab-paclitaxel plus gemcitabine. N Engl J Med. 2013;369:1691-703.

5. Rahma OE, Duffy A, Liewehr DJ, Steinberg SM, Greten TF. Second-line treatment in advanced pancreatic cancer: a comprehensive analysis of published clinical trials. Ann Oncol. 2013.

6. Cid-Arregui A, Juarez V. Perspectives in the treatment of pancreatic adenocarcinoma. World J Gastroenterol. 2015;21:9297-316.

7. Gresham GK, Wells GA, Gill S, Cameron C, Jonker DJ. Chemotherapy regimens for advanced pancreatic cancer: a systematic review and network meta-analysis. BMC Cancer. 2014;14:471.

8. Pelzer U, Schwaner I, Stieler J, Adler M, Seraphin J, Dorken B, et al. Best supportive care (BSC) versus oxaliplatin, folinic acid and 5-fluorouracil (OFF) plus BSC in patients for second-line advanced pancreatic cancer: a phase IIIstudy from the German CONKO-study group. Eur J Cancer. 2011;47:1676-81.

9. Oettle H, Riess H, Stieler JM, Heil G, Schwaner I, Seraphin J, et al. Second-line oxaliplatin, folinic acid, and fluorouracil versus folinic acid and fluorouracil alone for gemcitabine-refractory pancreatic cancer: outcomes from the CONKO-003 trial. J Clin Oncol. 2014;32:2423-9.

10. Wang-Gillam A, Li CP, Bodoky G, Dean A, Shan YS, Jameson G, et al. Nanoliposomal irinotecan with fluorouracil and folinic acid in metastatic pancreatic cancer after previous gemcitabine-based therapy (NAPOLI-1): a global, randomised, open-label, phase 3 trial. Lancet. 2015 Nov 29, [Epub ahead of print]

11. Kim ST, Choi YJ, Park KH, Oh SC, Seo JH, Shin SW, et al. A prognostic model to identify patients with advanced pancreas adenocarcinoma who could benefit from second-line chemotherapy. Clin Oncol (R Coll Radiol). 2012;24:105-11.

12. Lenzi R, Yalcin S, Evans DB, Abbruzzese JL. Phase II study of docetaxel in patients with pancreatic cancer previously untreated with cytotoxic chemotherapy. Cancer Invest. 2002;20:464-72.

13. Rougier P, Adenis A, Ducreux M, de Forni M, Bonneterre J, Dembak M, et al. A phase II study: docetaxel as first-line chemotherapy for advanced pancreatic adenocarcinoma. Eur J Cancer. 2000;36:1016-25.

14. Cereda S, Reni M. Weekly docetaxel as salvage therapy in patients with gemcitabine-refractory metastatic pancreatic cancer. J Chemother. 2008;20:509-12.

15. Saif MW, Syrigos K, Penney R, Kaley K. Docetaxel second-line therapy in patients with advanced pancreatic cancer: a retrospective study. Anticancer Res. 2010;30:2905-9.

16. Berk V, Ozdemir N, Ozkan M, Aksoy S, Turan N, Inal A, et al. XELOX vs. FOLFOX4 as second line chemotherapy in advanced pancreatic cancer. Hepatogastroenterology. 2012;59:2635-9.

17. El-Hadaad HA, Wahba HA. Oxaliplatin plus 5-fluorouracil and folinic acid (OFF) in gemcitabine-pretreated advanced pancreatic cancer: a phase II study. J Gastrointest Cancer. 2013;44:313-7.

18. Zaniboni A, Aitini E, Barni S, Ferrari D, Cascinu S, Catalano V, et al. FOLFIRI as second-line chemotherapy for advanced pancreatic cancer: a GISCAD multicenter phase II study. Cancer Chemother Pharmacol. 2012;69:1641-5.

19. Kouroussis C, Agelaki S, Mavroudis D, Kakolyris S, Androulakis N, Kalbakis K, et al. A dose escalation study of docetaxel and oxaliplatin combination in patients with metastatic breast and non-small cell lung cancer. Anticancer Res. 2003;23:785-91.

20. Raez LE, Santos ES, Lopes G, Rosado MF, Negret LM, Rocha-Lima C, et al. Efficacy and safety of oxaliplatin and docetaxel in patients with locally advanced and metastatic non-small-cell lung cancer (NSCLC). Lung Cancer. 2006:53:347-53.

21. Richards D, Kocs DM, Spira Al, David McCollum A, Diab S, Hecker LI, et al. Results of docetaxel plus oxaliplatin (DOCOX) +/- cetuximab in patients with metastatic gastric and/or gastroesophageal junction adenocarcinoma: results of a randomised Phase 2 study. Eur J Cancer. 2013;49:2823-31.

22. NCT00690300. Docetaxel plus Oxaliplatin as Therapy in Patients With Pancreatic Cancer. ClinicalTrials.gov 2008.

23. Simon R. Optimal two-stage designs for phase II clinical trials. Control Clin Trials. 1989;10:1-10.

24. Burris 3rd HA, Moore MJ, Andersen J, Green MR, Rothenberg ML, Modiano MR, et al. Improvements in survival and clinical benefit with gemcitabine as first-line therapy for patients with advanced pancreas cancer: a randomized trial. J Clin Oncol. 1997;15:2403-13. 
25. Bonnetain F, Dahan L, Maillard E, Ychou M, Mitry E, Hammel P, et al. Time until definitive quality of life score deterioration as a means of longitudinal analysis for treatment trials in patients with metastatic pancreatic adenocarcinoma. Eur J Cancer. 2010:46:2753-62.

26. Anota A, Hamidou Z, Paget-Bailly S, Chibaudel B, Bascoul-Mollevi C, Auquier $P$, et al. Time to health-related quality of life score deterioration as a modality of longitudinal analysis for health-related quality of life studies in oncology: do we need RECIST for quality of life to achieve standardization? Qual Life Res. 2015;24:5-18.

27. Ko AH, Tempero MA, Shan YS, Su WC, Lin YL, Dito E, et al. A multinational phase 2 study of nanoliposomal irinotecan sucrosofate (PEP02, MM-398) for patients with gemcitabine-refractory metastatic pancreatic cancer. Br J Cancer. 2013;109:920-5.

28. Yoo C, Hwang JY, Kim JE, Kim TW, Lee JS, Park DH, et al. A randomised phase II study of modified FOLFIRI.3 vs modified FOLFOX as second-line therapy in patients with gemcitabine-refractory advanced pancreatic cancer. Br J Cancer. 2009;101:1658-63.

29. Grothey A, Nikcevich DA, Sloan JA, Kugler JW, Silberstein PT, Dentchev T, et al. Intravenous calcium and magnesium for oxaliplatin-induced sensory neurotoxicity in adjuvant colon cancer: NCCTG N04C7. J Clin Oncol. 2011:29:421-7.

30. Loprinzi CL, Qin R, Dakhil SR, Fehrenbacher L, Flynn KA, Atherton P, et al, Phase III randomized, placebo-controlled, double-blind study of intravenous calcium and magnesium to prevent oxaliplatin-induced sensory neurotoxicity (N08CB/Alliance). J Clin Oncol. 2014;32:997-1005.

31. Hosein PJ, de Lima Lopes G, Jr., Pastorini VH, Gomez C, Macintyre J, Zayas G, et al. A phase II trial of nab-Paclitaxel as second-line therapy in patients with advanced pancreatic cancer. Am J Clin Oncol. 2013 Apr; 36(2):151-6.

32. Assaf E, Verlinde-Carvalho M, Delbaldo C, Grenier J, Sellam Z, Pouessel D, et al. 5-fluorouracil/leucovorin combined with irinotecan and oxaliplatin (FOLFIRINOX) as second-line chemotherapy in patients with metastatic pancreatic adenocarcinoma. Oncology. 2011;80:301-6.

33. Lee MG, Lee $\mathrm{SH}$, Lee SJ, Lee YS, Hwang JH, Ryu JK, et al. 5-Fluorouracil/ leucovorin combined with irinotecan and oxaliplatin (FOLFIRINOX) as second-line chemotherapy in patients with advanced pancreatic cancer who have progressed on gemcitabine-based therapy. Chemotherapy. 2013;59:273-9.

34. Soares HP, Bayraktar S, Blaya M, Lopes G, Merchan J, Macintyre J, et al. A phase II study of capecitabine plus docetaxel in gemcitabine-pretreated metastatic pancreatic cancer patients: CapTere. Cancer Chemother Pharmacol. 2014;73:839-45.

35. Xiong $H Q$, Varadhachary GR, Blais JC, Hess KR, Abbruzzese JL, Wolff RA Phase 2 trial of oxaliplatin plus capecitabine (XELOX) as second-line therapy for patients with advanced pancreatic cancer. Cancer. 2008;113:2046-52.

36. Demols A, Peeters M, Polus M, Marechal R, Gay F, Monsaert E, et al. Gemcitabine and oxaliplatin (GEMOX) in gemcitabine refractory advanced pancreatic adenocarcinoma: a phase II study. Br J Cancer. 2006;94:481-5.

37. Dragovich T, Laheru D, Dayyani F, Bolejack V, Smith L, Seng J, et al. Phase II trial of vatalanib in patients with advanced or metastatic pancreatic adenocarcinoma after first-line gemcitabine therapy (PCRT 04-001). Cancer Chemother Pharmacol. 2014;74:379-87.

\section{Submit your next manuscript to BioMed Central and we will help you at every step:}

- We accept pre-submission inquiries

- Our selector tool helps you to find the most relevant journal

- We provide round the clock customer support

- Convenient online submission

- Thorough peer review

- Inclusion in PubMed and all major indexing services

- Maximum visibility for your research

Submit your manuscript at www.biomedcentral.com/submit

) Biomed Central 